\title{
UNA SOCIEDAD PARA TODAS LAS EDADES: CURSO MONOGRÁFICO DE EJERCICIO FÍSICO
}

A SOCIETY FOR ALL AGES: MONOGRAPHIC COURSE OF PHYSICAL EXERCISE

\author{
Carmen Palmero ${ }^{1}$ \\ Isabel Luis $^{2}$ \\ M. Camino Escolar ${ }^{3}$ \\ Tamara de la Torre ${ }^{4}$ \\ Alfredo Jiménez ${ }^{5}$
}

\section{Resumen}

Los cambios de tendencia de la población (como mayor esperanza de vida o el aprendizaje a lo largo de la vida) ponen de manifiesto la necesidad de implementar políticas de intervención que se ajusten a las necesidades y las expectativas de las personas mayores. Con tal objetivo, se presenta el diseño de un curso monográfico de ejercicio físico para los alumnos mayores de 45 años de la Universidad Abierta de Personas Mayores de la Universidad de Burgos. La intervención se llevará a cabo a lo largo de un curso académico, y consta de 15 sesiones. Será conducido por estudiantes universitarios de los grados de Educación Social, Educación Infantil y Pedagogía, para fomentar de esta forma la cultura intergeneracional. Con el fin de valorar los resultados de la aplicación del programa de entrenamiento, se llevarán a cabo dos evaluaciones (pre-post intervención) con el Cuestionario de Salud SF-36, el cual valora la calidad de vida. Concluimos que numerosos estudios evidencian los beneficios saludables que reporta para este colectivo la práctica continuada del ejercicio físico, además de prevenir la dependencia física y aumentar la calidad de vida.

Palabras clave: personas mayores, universidad abierta, ejercicio físico, formación permanente, calidad de vida, ocio, jóvenes universitarios

\section{Abstract}

The population trend changes (e.g., increased life expectancy or life-long learning) highlight the need to implement intervention policies that fit the needs and expectations of older people. With that objective, this text presents the design of a monographic course of physical exercise for students over 45 years of the Universidad Abierta de Personas Mayores, in the University of Burgos. The intervention will take place over one academic year, and consists of 15 sessions. It will be led by university students in grades Social Education, Early Childhood Education and Pedagogy, thus promoting intergenerational culture. In order to assess the results of the implementation of this training program, two evaluations will be conducted (pre and post intervention), with SF-36 Health Survey, which evaluates the quality of life. We conclude that many studies show healthy benefits for this group after the continued practice of physical exercise, besides preventing dependency and increasing the quality of life.

Keywords: elderly people, open university, physical exercise, lifelong learning, qualily of living, leisure, university students

Fecha de recepción: 20 de noviembre de 2015

Fecha de aprobación: 4 de mayo de 2016

Para citar este artículo:

Palmero, C., Luis, I., Escolar, M. C., De la Torre, T. \& Jiménez, A. (2016). Una sociedad para todas las edades: curso monográfico de ejercicio físico. Lúdica Pedagógica, 24, 107-115.

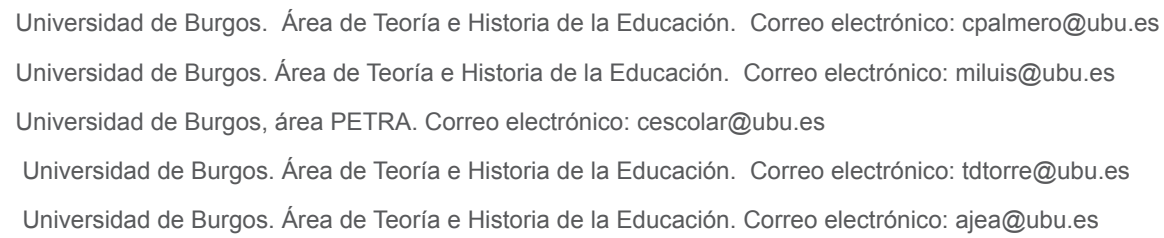




\section{INTRODUCCIÓN}

A partir del último tercio del siglo XX, España ha sufrido grandes cambios económicos y sociodemográficos, todos los cuales se han convertido en uno de los mayores retos a los que se enfrenta una sociedad desarrollada, como la nuestra (Crossette, 2011; De Diego Lázaro, 2010; Zaidi, Lelkes \& Borges, 2008). No pocas de estas transformaciones tienen como consecuencia una esperanza de vida cada vez más alta y, por ende, una población envejecida. Dicho incremento ha sido propiciado, entre otros factores, por los avances en el campo de la nutrición y por la mejora en las condiciones de vida; todo ello, combinado con los progresos que se han originado en las ciencias médicas (OMS, 2001; Rodríguez-Díez, 2015; Serrano, Oliva, París, Mendizábal, Prudenciano, Navas \& Plaza, 2013). Por otro lado, la actual situación de crisis económica, fruto de las políticas de consumo en el mundo entero, ha situado el envejecimiento poblacional como uno de las prioridades a las que las políticas sociales, sanitarias y económicas han de atender, por su impacto en la sociedad.

Por otro lado, también cabe tomar en cuenta que actualmente sufrimos un drástico descenso en las tasas de natalidad (Abades Porcel \& Rayon Valpuesta, 2012; De Diego Lázaro, 2010; Del Rey Poveda \& Ortega Osona, 2011). En tal sentido, el Instituto Nacional de Estadística, INE (2014), con predicciones de población de 2014-2064, pone de manifiesto que la tasa de nacimientos seguirá reduciéndose entre 2014 y 2028 , hasta alcanzar cifras del $24,8 \%$ menos que en los 15 años pasados. El porcentaje de población de más 65 años, que actualmente se sitúa en el 18,2\% de la población, pasará a ser del 24,9\% dentro de 15 años (2029), $y$ del $38,7 \%$ dentro de 50 años (en 2064).

Teniendo en cuenta las tendencias predictivas de la población, la tasa de dependencia (población menor de 16 años o mayor de 64 años) se elevará más de siete puntos, desde el $52,1 \%$ actual hasta el 59,2\% en 2029, y en 2064 alcanzaría el $95,6 \%$ de la población (INE, 2014).

Con estos datos, nos enfrentamos a un nuevo modelo de sociedad compuesto por sujetos de edades muy avanzadas (Abades Porcel \& Rayon Valpuesta, 2012), lo que se traduce en un impacto directo en la composición por edades de la población, al reducirse el número de personas de edades más jóvenes (Mirkin \& Weinberger, 2001).

Para hacer frente a este nuevo modelo y cubrir las necesidades y los intereses de las personas mayores, es necesario desarrollar nuevos proyectos que les permitan tener una percepción positiva de su estado de salud y participación social (Lázaro, 2009; Palmero \& Jiménez, 2008). De esta forma, podemos hablar de envejecimiento activo, concepto que fue adoptado por la Organización Mundial de la Salud, OMS, a finales de los años noventa del siglo XX, y con el cual se logra que la persona desarrolle un bienestar físico, social y mental mediante la participación en la sociedad de acuerdo con sus necesidades, sus deseos y sus capacidades, y proporcionándoles, a su vez, protección, seguridad y los cuidados adecuados (OMS, 2002).

Con el fin de que los impactos sociales, sanitarios y económicos que acarrea una población envejecida sean sostenibles, se han llevado a cabo distintas iniciativas para personas mayores, teniendo en cuenta sus necesidades y sus intereses, para que se conviertan en sujetos activos. Entre las distintas áreas de intervención encontramos el cuidado activo de salud, la prevención y la educación socio-sanitaria, programas de formación socioeducativa y programas de cultura, ocio y tiempo libre, entre otros, todos los cuales se enmarcan dentro las políticas de envejecimiento activo que orientan la acción (Botero de Mejía, Merchán \& Eugenia, 2007; López Fraga, 2013).

Este artículo se centra en el cuidado activo de la salud, término con el cual nos referimos a la práctica de ejercicio físico, yoga, memoria y psicomotricidad, como una forma activa de envejecer favoreciendo el desarrollo de las diversas dimensiones de la persona y mejorando así su calidad de vida (Plan de Acción para la Promoción del Envejecimiento Activo, 2010).

Envejecer conlleva una serie de modificaciones en el organismo, caracterizada, en ocasiones, por una disminución de las cualidades físicas y de la capacidad funcional, lo que, a su vez, puede llevar a una reducción de las actividades cotidianas y, con ello, obligar a una situación de dependencia (Carazo Vargas, 2001; Verdugo, Gómez \& Arias, 2009). Además, según Hamer, Batty, Stamatakis y Kivimaki (2011) y Zimmer, Martin, Jones y Nagin (2014), una reducida funcionalidad física en este grupo poblacional está directamente asociada a un descenso en la calidad de vida, incluido un mayor riesgo de demencia y de mortalidad (Marcos Pardo, 2014).

El ejercicio físico es un factor clave del estilo de vida que tiene efectos beneficiosos sobre la longevidad, pues favorece la condición física, la capacidad funcional y tiene un impacto preventivo en el desarrollo de enfermedades degenerativas (García, Baeza \& Fernández, 2010; González Jurado, 2004; Varela Moreiras \& Silvestre Castelló, 2010). 
Tal como señalan diversos autores (Audelin, Savage \& Ades, 2008; Owen \& Croucher, 2000), la práctica del ejercicio físico reduce la incidencia de algunas enfermedades cardiovasculares, mediante la disminución y la prevención de los factores de riesgo asociados. Continuando en esta línea, Hunter, McCarthy y Bamman (2004) y Phillips (2007) exponen que su práctica conlleva un fortalecimiento muscular que trae consigo un menor riesgo de sufrir fracturas y caídas, así como la mejora del equilibrio y de la agilidad que presentan las personas sedentarias (Martínez, Cocca, Mohamed \& Ramírez, 2010). En esta línea, Bruce, Fries y Lubeck (2007) señalan que, igualmente, se produce una reducción del dolor musculoesquelético asociado al envejecimiento.

Los programas de ejercicio físico (Brannon \& Feist, 2001; Meléndez, Tomás, Oliver \& Navarro, 2009; Sánchez, Ureña \& Garcés de los Fayos, 2002) mejoran el bienestar físico y psíquico, la autoestima, la satisfacción con la vida y la forma de enfrentarse a esta. Sonstroem (1984) señala que la mejora de la autoestima a través de la actividad física reside en que las personas se sienten mejor físicamente y desarrollan nuevas experiencias sociales con sus compañeros, y así se favorecen la cohesión y la integración social de la persona (citado en Remor \& Martins de Lima, 2009).

En tal sentido, diversos estudios, como los de Ossip-Klein et al (1989), han demostrado que la práctica de actividad física genera un aumento en la liberación de endorfinas, lo cual produce una mejora del estado del ánimo, y, por ende, optimismo y bienestar.

Para Buffone (1984, citado en Guillén García, F., Castro Sánchez \& Guillén García, M. A., 2003), el ejercicio físico mejora el humor, pues favorece las funciones cognitivas. Por otra parte, existe una relación inversa entre la práctica de ejercicio físico y la aparición de síntomas relacionados con la ansiedad y la depresión (Martínez et al., 2010); a su vez, el ejercicio físico protege frente al riesgo de desarrollar demencia o Alzheimer. También aumenta la productividad, lo cual hace que los sujetos estén más activos y aprovechen el día (García et al., 2010).

Dentro de este contexto, muchas han sido las universidades que apuestan por la formación a lo largo de la vida, y por ello cuentan con programas dirigidos a personas mayores de 45 años. La finalidad principal de estos programas es prevenir situaciones de dependencia, promocionar la autonomía personal y mejorar la calidad de vida. Se busca motivar a las personas adultas para que ejerzan una ciudadanía participativa dotándolas de los conocimientos, las destrezas, las habilidades y las competencias que les sirvan como fórmula de crecimiento personal y social (Cuenca Cabeza, 2004; March Cerdá, 2008). Esta formación no profesionalizante permite que el alumnado haga frente a los diversos retos que conlleva la edad, les ayuda a expresarse física, mental $\mathrm{y}$ emocionalmente, y a que sientan que tienen control de sus propias vidas y son útiles; enmarcada dentro de los programas de envejecimiento activo, dicha formación pretende brindarles la posibilidad a las personas mayores de desarrollar un ocio formativo, como vía para promover la participación social, pues el aumento de la participación forma parte del ciclo del bienestar a medida que se envejece (Guerrero Romera, 2002).

Dentro de esta nueva realidad social, se plantea la necesidad de articular formas de convivencia entre generaciones, ya que en una sociedad más longeva las distintas generaciones deben convivir más tiempo. Por ello, las relaciones intergeneracionales han adquirido una especial relevancia en el contexto de la atención a las personas mayores, y, por ende, también ha sido así en la planificación de los Programas Universitarios de Personas Mayores: así, ya en 1999, Año Internacional de las Personas Mayores, se estableció como lema "Una sociedad para todas las edades" (COM, 221; COM, 94), lo cual se convierte en principio determinante del envejecimiento activo (Kaleche, Barreto \& Keller, 2005; Walker, 2006).

Estudios previos sobre el impacto de los Programas Universitarios para Mayores han demostrado que la participación en estos se correlaciona positivamente con el hecho de vivir una jubilación satisfactoria y gozar de una buena calidad de vida. Existen beneficios relacionados con la salud mental originados por actividades intelectuales, lo que redunda en un retraso en la aparición de problemas cognitivos o de demencias. Igualmente, contribuyen a desarrollar habilidades sociales, al relacionarse con nuevas personas (Villaplana Prieto, 2010).

Por lo anterior, el objetivo de este artículo es presentar el diseño de un programa monográfico de ejercicio físico para los alumnos matriculados en la Universidad Abierta de Personas Mayores de la Universidad de Burgos. Las clases serán conducidas por estudiantes de 
los grados de Educación Social, Maestros de Educación Primaria y Pedagogía, como forma de contribuir, desde un ocio formativo en calidad de vehículo de aprendizaje, al aumento del bienestar psicológico, físico y social de las personas mayores, además de lograr un cambio de percepción por parte de los jóvenes sobre este colectivo (Bermejo García, 2010; Cabedo \& Escuder-Mollón, 2014; Gutiérrez Sánchez \& Hernández Torrano, 2013; Pérez Serrano \& De Juanas Oliva, 2014).

\section{DISEÑO DE LA PROPUESTA DE INTERVENCIÓN}

\section{Justificación}

La finalidad de este proyecto de intervención es que la persona mayor adquiera un conjunto de habilidades y destrezas mediante el ejercicio físico, con el fin de disminuir la probabilidad de sufrir una futura dependencia, así como mejorar su calidad de vida y fomentar la integración social. Los objetivos propuestos nacen de la importancia que adquiere el ejercicio físico en todas las potencialidades de la persona en los ámbitos motor, cognitivo y afectivo. La decisión de desarrollar esta propuesta de intervención dentro de la Universidad Abierta para Mayores de la Universidad de Burgos (UBU) surge porque no existe ninguna formación similar.

\section{Destinatarios}

Este programa va dirigido a personas mayores de 45 años inscritas como alumnos de la Universidad Abierta para mayores (UA) de la Universidad de Burgos. El perfil del alumnado potencial de la Universidad Abierta tiende a ser femenino, con edades comprendidas entre los 55 y los 75 años, residente en Burgos y provincia. Su situación laboral es de jubilado o prejubilado, con gran disposición y tiempo libre, además con necesidad de cubrir este tiempo libre. El nivel de estudios es variado, si bien destacan en su mayoría los estudios primarios, la formación profesional y las diplomaturas universitarias. En cuanto a su salud física y emocional, esta, en general, es buena.

\section{Objetivos}

1. Inculcar la importancia del ejercicio físico en el colectivo de mayores.

2. Fomentar la participación de las personas mayores en actividades físicas.
3. Eliminar prejuicios y estereotipos de los jóvenes hacia las personas mayores, tan solo por su edad, a través de las actividades intergeneracionales.

4. Determinar si las características físicas y psicológicas mejoran tras la implementación del programa.

5. Comprobar si la percepción de la calidad de vida mejora con el programa de ejercicio físico.

6. Fomentar un ocio saludable e intergeneracional en los alumnos universitarios de diferentes grados de la UBU al ser los responsables de conducir este programa para personas mayores.

Metodología

El método de trabajo es eminentemente práctico, acompañando de explicaciones teóricas sobre el beneficio de la ejecución de la actividad.

\section{Cronograma}

La intervención se llevará a cabo a lo largo de un curso académico, y abarcará de octubre a febrero; es decir, a lo largo de cinco meses, de forma ininterrumpida.

Esta acción formativa de ejercicio físico está dividida en 15 sesiones, las cuales se impartirán todos los lunes durante una hora.

Recursos

Para desarrollar, aplicar y coordinar la intervención educativa, contamos con el alumnado de prácticas de los grados de Educación Social y Pedagogía. Ello, tomando en cuenta que dentro de las competencias que han de adquirir a lo largo de su travesía académica estos futuros profesionales está diagnosticar las necesidades y las posibilidades de desarrollo de las personas mayores, para así fundamentar las acciones educativas pertinentes que favorezcan el desarrollo de estrategias y de técnicas para promover la participación y el aprendizaje a lo largo de la vida.

Además, los alumnos de ambos grados han de desarrollar la capacidad de integrar los conocimientos teóricos relacionados con cada momento del proceso de elaboración y gestión de programas: análisis de la realidad, planificación, puesta en marcha, evaluación de la inter- 
vención educativa. Por todo ello, son un recurso humano fundamental dentro del programa de ejercicio físico, como figura de mediación y dinamización.

En relación con los recursos materiales, se cuenta, respecto a infraestructura física, con el Polideportivo de la Universidad de Burgos y la Piscina Municipal San Amaro.

\section{Actividades}

En la tabla 1 se muestran todas las actividades previstas para cada una de las sesiones.

Tabla 1. Actividades y sesiones

\begin{tabular}{|c|l|l|l|}
\hline Sesión & Parte inicial & Parte principal & Parte final \\
\hline 1 & Calentamiento & Espalda sana & Relajación \\
\hline 2 & Calentamiento & Flexibilidad & Estiramiento \\
\hline 3 & Calentamiento & Resistencia & Juegos \\
\hline 5 & Calentamiento & Fuerza muscular & Estiramiento \\
\hline 6 & Calentamiento & $\begin{array}{l}\text { Natación / } \\
\text { Espalda sana }\end{array}$ & Relajación \\
\hline 7 & Calentamiento & $\begin{array}{l}\text { Fortalecimiento } \\
\text { muscular II }\end{array}$ & Estiramiento \\
\hline 8 & & $\begin{array}{l}\text { Sesión } \\
\text { Intergeneracional }\end{array}$ & \\
\hline 9 & & $\begin{array}{l}\text { Sesión } \\
\text { Intergeneracional }\end{array}$ & \\
\hline 10 & & $\begin{array}{l}\text { Sesión } \\
\text { Intergeneracional }\end{array}$ & \\
\hline 11 & & Memoria & \\
\hline 12 & & Baile & Baile \\
\hline 13 & & $\begin{array}{l}\text { Juegos } \\
\text { cooperativos }\end{array}$ & $\begin{array}{l}\text { Terapia corporal: } \\
\text { Risoterapia }\end{array}$ \\
\hline 14 & & equilibrio/Pilates & \\
\hline 15 & & & \\
\hline
\end{tabular}

Fuente: elaboración propia.

En todas las sesiones se deberá realizar un calentamiento previo al ejercicio. Se exceptúa la sesión 5 (natación), puesto que tiene un calentamiento inicial propio; también, las sesiones 11 y 12 (danza) y 15 (terapia corporal: risoterapia).

\section{Instrumentos de evaluación}

Para evaluar los resultados de la intervención desarrollada, se aplicará a los alumnos participantes en el programa el cuestionario de Salud SF-36, sobre calidad de vida. Se trata de un instrumento genérico de medición de calidad de vida relacionada con salud, y el cual consta de 36 ítems que abarcan las siguientes escalas: función física, rol físico, salud general, vitalidad, función social, rol emocional y salud mental. Por último, incluye un ítem de transición de salud que pregunta sobre el cambio en el estado de salud general respecto al año anterior.

Las escalas del SF-36 están ordenadas de forma que a mayor puntuación, mejor es el estado de salud (Vilagut et al., 2005). La tabla 2 contiene el número de ítems de las diferentes escalas del cuestionario y una descripción del significado de las puntuaciones altas y bajas. Para el cálculo de las puntuaciones, después de pasar el cuestionario es necesario: a) homogeneizar la dirección de las respuestas mediante la recodificación de los diez ítems que lo requieran, para que todos los ítems sigan el gradiente de "a mayor puntuación, mejor estado de salud"; b) calcular el sumatorio de los ítems que componen la escala (puntuación cruda de la escala), y c) transformar de forma lineal las puntuaciones crudas para obtener puntuaciones en una escala entre 0 y 100 (puntuaciones transformadas de la escala) (Vilagut et al., 2005).

Así pues, para cada dimensión, los ítems son codificados, agregados y transformados en una escala que tiene un recorrido desde 0 (el peor estado de salud para esa dimensión) hasta 100 (el mejor estado de salud) (tabla 2). En caso de que falte información, si se han contestado al menos el 50\% de los ítems de una escala, se recomienda sustituir cualquier ítem ausente por el promedio de los ítems completados de esta. En caso contrario (más del 50\% de ítems no contestados), la puntuación de dicha escala no se debería calcular. Además, el cuestionario permite el cálculo de dos puntuaciones sumario, la componente sumario física (PCS) y la mental (MCS), mediante la combinación de las puntuaciones de cada dimensión (Vilagut et al., 2005).

El SF-36 proporciona un perfil del estado de salud y es aplicable tanto a los pacientes como a la población sana. Está dirigido a personas de 14 o más años de edad; preferentemente, debe ser autoadministrado, aunque también es aceptable su administración por medio de entrevista personal o telefónica (Arostegui Madariaga \& Nuñez-Antón, 2008).

Se han publicado diversos estudios de validación del SF-36, que lo comparan con otros instrumentos de medición de salud más generales y complejos (Brazier et al., 1992; Stansfeld, Roberts \& Foot, 1997). El cuestionario SF-36 ha sido traducido en más de 50 países como parte del International Quality of Life Assessment 
(IQOLA) Project (Aaronson et al., 1992; Gandek \& Ware, 1998). Este proyecto incluye a investigadores de 14 países, incluyendo España. Las adaptaciones validadas y publicadas por el proyecto IQOLA incluyen la versión en castellano (Alonso, Prieto \& Antón, 1995). Una década después de su creación, Vilagut et al. (2005) han hecho una revisión crítica del contenido, las propiedades métricas y los nuevos desarrollos de la versión castellana del SF-36.

Tabla 2. Contenido de las escalas del SF-36

\begin{tabular}{|c|c|c|c|}
\hline Dimensión & № ítems & Peor puntuación (0) & Mejor puntuación (100) \\
\hline Función física & 10 & $\begin{array}{l}\text { Muy limitado para llevar a cabo todas las activida- } \\
\text { des físicas, debido a la salud (bañarse, ducharse) }\end{array}$ & $\begin{array}{l}\text { Lleva a cabo todo tipo de actividad física sin nin- } \\
\text { guna limitación }\end{array}$ \\
\hline Rol físico & 4 & $\begin{array}{l}\text { Problemas con el trabajo u otras actividades } \\
\text { diarias, debido a la actividad física }\end{array}$ & $\begin{array}{l}\text { Ningún problema con el trabajo u otras actividades } \\
\text { diarias }\end{array}$ \\
\hline Dolor corporal & 2 & Dolor muy intenso y extremadamente limitante & Ningún dolor ni limitación \\
\hline Salud general & 5 & $\begin{array}{l}\text { Evalúa como mala la propia salud y cree posible } \\
\text { que empeore. }\end{array}$ & Evalúa la propia salud como excelente \\
\hline Vitalidad & 4 & Se siente cansado y exhausto todo el tiempo & $\begin{array}{l}\text { Se siente muy dinámico y lleno de energía todo el } \\
\text { tiempo }\end{array}$ \\
\hline Función social & 2 & Interfiere de forma extrema con las actividades & Lleva a cabo actividades sociales normales \\
\hline Rol emocional & 3 & $\begin{array}{l}\text { Problemas con el trabajo y otras actividades } \\
\text { diarias, debido a problemas emocionales }\end{array}$ & Ningún problema con el trabajo y otras actividades \\
\hline Salud mental & 5 & $\begin{array}{l}\text { Sentimientos de angustia y depresión durante } \\
\text { todo el tiempo }\end{array}$ & $\begin{array}{l}\text { Sentimiento de felicidad, tranquilidad y calma } \\
\text { durante todo el tiempo }\end{array}$ \\
\hline $\begin{array}{l}\text { Ítem de transi- } \\
\text { ción de salud }\end{array}$ & 1 & $\begin{array}{l}\text { Cree que su salud es mucho peor ahora que hace } \\
\text { un año. }\end{array}$ & $\begin{array}{l}\text { Cree que su salud general es mucho mejor ahora } \\
\text { que hace un año. }\end{array}$ \\
\hline
\end{tabular}

Fuente: extraído y adaptado de Vilagut, G., Ferrer, M., Rajmil, L., Rebollo, P., Permanyer-Miralda, G., Quintana, J. M., ... Alonso, J. (2005). El Cuestionario de Salud SF-36 español: una década de experiencia y nuevos desarrollos. Gaceta Sanitaria, 19 (2).

Finalmente, se prevén dos momentos de recogida de datos: uno de preintervención y otro de posintervención. Para el análisis de los resultados, se utilizará el Programa Estadístico IBM SPSS. Esta evaluación permitirá comprobar si las personas tras la intervención (es decir, tras la práctica continuada de ejercicio físico) se perciben mejor tanto física como psicológicamente.

\section{CONCLUSIONES}

Tras el estudio preliminar efectuado en la parte teórica, podemos apuntar el beneficio que se obtiene de forma tanto individual como social al practicar ejercicio físico; máxime, en relación con las personas mayores, pues los procesos implicados en el envejecimiento se ven limitados, lo que permite niveles óptimos de calidad de vida.

Por otro lado, el ocio formativo intergeneracional se relaciona con la práctica de la ciudadanía activa, por lo que, al presentar el diseño de un programa monográfico de ejercicio físico para las personas mayores matricula- das en la Universidad Abierta de la UBU, conducido por estudiantes en prácticas de los grados universitarios de Educación Social, Maestro en Educación Primaria y Pedagogía, aunamos ambas perspectivas, para dotarlas de justificación científica. Por otra parte, las sesiones intergeneracionales, que se llevarán a cabo en el programa entre los alumnos receptores (personas mayores de 45 años) y los alumnos en prácticas de grados universitarios, en su calidad de conductores del programa, tienen como finalidad la participación conjunta de ambos rangos generacionales, de forma que se promueva un cambio en las visiones estereotipadas sobre las capacidades de las personas mayores, y así disminuyan los prejuicios.

Como se ha recogido en el presente artículo, la realidad actual de este colectivo plantea la necesidad de aplicar otra forma de intervención socioeducativa, con la perspectiva de sus posibilidades. De ahí la importancia de un envejecimiento activo, con el que las personas se sientan autónomas, participativas e independientes. El proceso de envejecimiento activo requiere potenciar condicio- 
nes favorables para llevar una vida participativa, con salud y calidad de vida; para ello, hay que incidir en las variables predictoras, y un factor clave al respecto es el ejercicio físico.

\section{REFERENCIAS}

Abades Porcel, M. \& Rayon Valpuesta, E. (2012). El envejecimiento en España: ¿un reto o problema social? Gerokomos, 23 (4), 151-155. https://dx.doi. org/10.4321/S1134-928X2012000400002

Aaronson, N. K., Acquadro, C., Alonso, J., Apolone, G., Bucquet, D., Bullinger, M., Bungay, K., Fukuhara, S., Gandek, B., Keller, S., Razavi, D., Sanson-Fisher, R., Sullivan, M., Wood-Dauphinee, S., Wagner, A. \& Ware, J. E. (1992). International quality of life assessment (IQOLA) project. Quality of Life Research, 1 (5), 349351.

Alonso, J., Prieto, L. \& Antón, J. M. (1995). La versión española del SF-36 Health Survey (Cuestionario de Salud SF-36): Un instrumento para la medida de los resultados clínicos. Medicina Clínica, 104 (20), 771776.

Arostegui Madariaga, I. \& Nuñez-Antón, V. (2008). Aspectos estadísticos del Cuestionario de Calidad de Vida relacionada con salud Short Form-36 (SF-36). Estadística Española, 50 (167), 147-192.

Audelin, M. C., Savage, P. D. \& Ades, P. A. (2008). Changing clinical profile of patients entering cardiac rehabilitation/secondary prevention programs: 1996 to 2006. Journal of cardiopulmonary rehabilitation and prevention, 28 (5), 299-306.

Brannon, L. \& Feist, J. (2001). Psicología de la salud. Madrid: Thomson-Paraninfo.

Brazier, J. E., Harper, R., Jones, N. M. B., O'Cathain, A., Thomas, K.J., Usherwood, T., \& Westlake, L. (1992). Validating the SF-36 Health Survey questionnaire: New outcome measure for primary care. British Medical Journal, 305, 160-164.

Bermejo García, L. (2010). Envejecimiento activo y actividades socioeducativas con personas mayores. Madrid: Editorial Médica Panamericana.

Botero de Mejía, B. E., Merchán, P. \& Eugenia, M. (2007). Calidad de vida relacionada con la salud (CVRS) en adultos mayores de 60 años: una aproximación teórica. Hacia la Promoción de la Salud, 12 (1), 11-24.

Bruce, B., Fries, J. F. \& Lubeck, D. P. (2007). Aerobic exercise and its impact on musculoskeletal pain in older adults: a 14 year prospective, longitudinal study. Arthritis Research \& Therapy, 7 (6), 263-270.
Carazo Vargas, P. (2001). Actividad física y capacidad funcional en el adulto mayor: el taekwondo como alternativa de mejoramiento. Revista Educación, 25 (2), 125-135.

Cabedo, S. \& Escuder-Mollón, P. (2014). Educación y calidad de vida en personas mayores. Castelló de la Plana: UNE.

Crossette, B. (2011). El estado de la población mundial 2011. UNFPA.

COM (1999) 221: Hacia una Europa de todas las edades.

COM (2005) 94: Confronting Demographic Change: a new solidarity between generations.

Cuenca Cabeza, M. (2004). Pedagogía del ocio: Modelos y propuestas. Bilbao: Universidad de Deusto.

De Diego Lázaro, E. (2010). La población española actual. Comportamiento demográfico. La incidencia de los movimientos migratorios y sus consecuencias. Proyecto Clío 36. Recuperado de http://clio.rediris.es

Del Rey Poveda, A. \& Ortega Osona, J. A. (2011). La preproducción de la población en las provincias españolas (1975-2005). Revista internacional de sociología (RIS), 69, 91-120.

Gandek, B. \& Ware, J. E. (1998). Methods for validating and norming translations of health status questionnaires: The IQOLA project approach. Journal of Clinical Epidemiology, 51 (11), 953-959.

García, V. A., Baeza, A. C. \& Fernández, M. D. (2010). Beneficios de la actividad física en personas mayores. Revista Internacional de Medicina y Ciencias de la Actividad Física y del Deporte, 40, 4-20.

Guerrero Romera, C. (2002). Evaluación de los programas universitarios para mayores: autopercepción de los alumnos. España: Enlace en red.

Guillén García, F., Castro Sánchez, J. J. \& Guillén García, M. A. (2003) Calidad de vida, salud y ejercicio físico: una aproximación al tema desde una perspectiva psicosocial. Revista de psicología del deporte, 68 (12), 91-107.

González Jurado, J. (2004). La actividad física orientada a la promoción de la salud. Escuela abierta, 45 (7), 7396.

Gutiérrez Sánchez, M. \& Hernández Torrano, D. (2013). Los beneficios de los programas intergeneracionales desde la perspectiva de los profesionales. Pedagogía Social. Revista Interuniversitaria, 22, 133-146.

Hamer, M., Batty, G. D., Stamatakis, E. \& Kivimaki, M. (2011). Comparison of risk factors for fatal stroke and ischemic heart disease: A prospective follow up of the health survey for England. Atherosclerosis, 219 (2), 807-810. 
Hunter, G. R., McCarthy,J.P.\& Bamman, M. M. (2004). Effects of resistance training on older adults. Sports Med, 34 (5), 329-348.

Instituto Nacional de Estadística, INE (2014). Proyecto de población en España 2014-2064.

Kaleche, A., Barreto, S.M. \& Keller, I. (2005): “Global Ageing: The demographic revolution in all cultures and societies". En M. L. Johnson (Ed.), The Cambridge handbook of age and ageing (pp. 30-46). Cambridge: Cambridge University Press.

Lázaro, Y. (2009). Aprender disfrutando: una experiencia de ocio para adultos/mayores en la Universidad. Revista Mal Estar e Subjetividade, 9 (3), 751- 782.

López Fraga, A. (2013). Envejecimiento exitoso en función de la percepción del nivel de salud en personas mayores que participan en programas de envejecimiento activo. Revista terapia ocupacional, 10 (17), 20. Recuperado de: http://www.revistog.com/num17/pdfs/revision. pdf.

March Cerdá, M. X. (2008). La formación universitaria de personas mayores y la promoción de la autonomía personal en la unión europea. En Formación universitaria de personas mayores y promoción de la autonomía personal. Políticas socioeducativas, metodologías e innovaciones, 47-68. Burgos: Servicio de publicaciones de la Universidad de Burgos.

Marcos Pardo, P. J. (2014) Motivación autodeterminada en adultos mayores practicantes de ejercicio físico. Cuaderno de psicología del deporte, 14 (3), 149-156.

Martínez, F., Cocca, A., Mohamed, K., \& Ramírez, J. (2010). Actividad física y sedentarismo: Repercusiones sobre la salud y calidad de vida de las personas mayores. Retos. Nuevas tendencias en Educación Física, Deporte y Recreación, 17, 126-129.

Meléndez, J. C., Tomás, J. M., Oliver, A. \& Navarro, E. (2009). Psychological and physical dimensions explaining life satisfaction among the elderly: A structural model examination. Archives of Gerontology and Geriatrics, 48 (3), 291-295.

Mirkin, B. \& Weinberger, B. (2001). The demography of population ageing. Population Bulletin of the United Nations (42/43), 37-53.

Organización Mundial de la Salud, OMS (2001). Abrazo Mundial. Manual. Recuperado de http://www.who. int/ageing/publications/alc_elmanual.pdf

Organización Mundial de la Salud, OMS (2002). Envejecimiento activo: un marco político. Revista Española Geriatría Gerontológica (S2), 74-105.

Ossip-Klein, D. J., Doyne, E. J., Bowman, E. D., Osborn, K. M., McDougall-Wilson, I. B. \& Neimeyer,
R. A. (1989). Effects of running or weight lifting on self-concept in clinically depressed women. J Consult Clin Psychol, 57 (1), 158-61.

Owen, A. \& Croucher, L. (2000). Effect of an exercise programme for elderly patients with heart failure. European journal of heart failure, 2 (1), 65-70.

Palmero, C. \& Jiménez, A. (2008). The quality of university programs for older people in Spain: Innovations, tendencies and ethics in the face of active ageing and the European Higher Education Area. Educational Gerontology, 44 (4).

Pérez Serrano, G. \& De Juanas Oliva, A. (2014). Educación y jóvenes en tiempos de cambio. Madrid: UNED.

Phillips, S. M. (2007) Resistance exercise: good for more than just Grandma and Grandpa's muscles. Appl. Physiol. Nutr. Myab, 32, 1198-1205.

Plan de Acción para la Promoción del envejecimiento activo en Bizkaia (2010-2011). Bilbao: Diputación Foral de Bizkaia. Recuperado de http://www.bizkaia. eus/home2/Archivos/DPT03/Temas/Pdf/Plan\%20 de $\% 20$ actuaci $\%$ C 3\%B3n $\% 20$ en $\% 20$ materia $\% 20$ de\%20envejecimiento\%20activo.pdf

Rodríguez-Díez, J. L. (2015). Repercusión de la formación en adultos mayores: El caso del Aula Abierta de Mayores. Pedagogía social. Revista interuniversitaria, 26, 372-374.

Remor, E. \& Martins de Lima, S. (2009). Efecto diferencial del componente lúdico dentro de un programa específico de ejercicio físico para personas mayores sobre la autoestima, autoeficacia y satisfacción con la vida. Universidad Autónoma de Madrid, 4 (2), 233-252.

Sánchez, P., Ureña, F. \& Garcés de los Fayos, E. J. (2002). Repercusiones de un programa de actitud física gerontológico sobre la actividad física, autoestima, depresión y afectividad. Cuadernos de psicología del deporte, 2 (2), 57-73.

Serrano, G. P., Oliva, Á. D. J., París, E. C., Mendizábal, M. R. L., Prudenciano, J. L., Navas, M. D. C. O. \& Plaza, Á. M. (2013). Calidad de vida en personas adultas y mayores. Madrid: UNED.

Sonstroem, R. J. (1984). Exercise and self-esteem. Exercise and Sport Sciences Reviews, 12, 123-156.

Stansfeld, S. A., Roberts, R. \& Foot, S. P. (1997). Assessing the validity of the SF-36 general Health Survey. Quality of Life Research, 6 (3), 217-224.

Varela Moreiras, G. \& Silvestre Castelló, D. (2010). Relación actividad física y estado de salud en la menopausia y el envejecimiento. Nutrición, vida activa y deporte. Madrid: Universidad de San Pablo. 
Verdugo, M. A., Gómez, L. E. \& Arias, B. (2009). La Escala FUMAT de evaluación de la calidad de vida de personas mayores y con discapacidad. Salamanca: Universidad de Salamanca, Publicaciones del INICO, Colección Herramientas, 4.

Vilagut, G., Ferrer, M., Rajmil, L., Rebollo, P., PermanyerMiralda, G., Quintana, J. M., Santed, R., Valderas, J. M., Ribera, A., Domingo-Salvany, A. \& Alonso, J. (2005). El cuestionario de salud SF-36 español: Una década de experiencia y nuevos desarrollos. Gaceta Sanitaria, 19 (2), 135-150.

Villaplana Prieto, C. (2010). Relación entre los Programas Universitarios para Mayores, la satisfacción durante la jubilación y la calidad de vida. Revista de Investigación Educativa, 28 (1), 195-216.
Walker, A. (2006). Active ageing in employment: Its meaning and potential. Asia-Pacific Review, 13 (1), 7893.

Zaidi, A., Lelkes, O. \& Borges, I. (2008). Documentos internacionales. Envejecimiento de la población europea: la felicidad, la protección de la dignidad y el envejecimiento saludable. (Trad. Calleja Miranda, J). Boletín sobre envejecimiento, 37. IMSERSO.

Zimmer, Z., Martin, L. G., Jones, B. L. \& Nagin, D. S. (2014). Examining late-life functional limitation trajectories and their associations with underlying onset, recovery, and mortality. The Journals of Gerontology Series B: Psychological Sciences and Social Sciences, 69 (2), 275286. 